\title{
The influence of multiple readings on the missing-letter effect revisited
}

\author{
Jean Saint-Aubin ANd ANNIE Roy-Charland \\ Université de Moncton, Moncton, New Brunswick, Canada \\ AND \\ RAYMOND M. KLEIN \\ Dalhousie University, Halifax, Nova Scotia, Canada
}

\begin{abstract}
In searching for a target letter while reading, participants make more omissions when the target letter is embedded in frequent function words than when it is embedded in less frequent content words. According to the guidance-organization (GO) model, this occurs because high-frequency function words are processed faster than low-frequency content words, leaving less time available for letter processing. We tested this hypothesis in three experiments by increasing word-processing speed through text repetition, which should translate into higher omission rates. Participants either read the text and searched for the target letter once or read the text three times and searched for a target letter on all readings or the final reading only. In all the experiments in which participants could not anticipate the target letter to be used, results revealed the presence of a large missingletter effect that was unaffected by familiarity with the text. In addition, when participants knew from the start the target letter to be used on the final reading, the missing-letter effect was eliminated. Repeated search of the same text for different targets increased omissions equally for function words and content words, but this finding was present even when a new text was used, suggesting that repetition of the search task, rather than familiarity with the text, was responsible.
\end{abstract}

When readers are asked to search for a target letter while reading a prose passage for comprehension, they miss more target letters embedded in frequent function words than those embedded in less frequent content words (Corcoran, 1966; Healy, 1994; Koriat \& Greenberg, 1994; SaintAubin \& Poirier, 1997). This well-replicated phenomenon, known as the missing-letter effect, has been extensively investigated over the last three decades as a window on the psycholinguistic representations and processes involved in reading (Healy, 1994). The missing-letter effect has been found to be sensitive to a wide range of factors with wellknown influences on reading processes. In effect, the size of the missing-letter effect is influenced by word frequency (see, e.g., Assink, van Well, \& Knuijt, 2003; Moravcsik \& Healy, 1995; Roy-Charland \& Saint-Aubin, 2006; SaintAubin \& Klein, 2004), word function (see, e.g., Koriat \& Greenberg, 1991; Saint-Aubin \& Poirier, 1997), eye movement patterns (Saint-Aubin \& Klein, 2001), text font and viewing conditions (Drewnowski \& Healy, 1977; Healy, Oliver, \& McNamara, 1987; Saint-Aubin, Klein, \& RoyCharland, 2003; Schneider \& Healy, 1993), target letter position within a word (Assink \& Knuijt, 2000), prosody and stress pattern (see, e.g., Mohan, 1978; Read, 1983; Schneider, Healy, \& Gesi, 1991), reading skills and reading development (see, e.g., Cunningham, Healy, Kanen- giser, Chizzick, \& Willitts, 1988; Drewnowski, 1978, 1981; Mohan, 1978; Saint-Aubin, Klein, \& Landry, 2005), and text repetition (Greenberg \& Tai, 2001). The present article is centered on text repetition, since its influence on the missing-letter effect is still unclear and constitutes a challenge for models of the effect.

Over the last two decades, the missing-letter effect has benefited from a vigorous and fruitful theoretical debate between the two leading models aimed at accounting for this effect (see, e.g., Healy, 1994; Koriat \& Greenberg, 1994). Recently, the debate resumed with the development of a unifying framework called the guidance-organization (GO) model, which retains the major assumptions of the previous two competing models (Greenberg, Healy, Koriat, \& Kreiner, 2004). The GO model can be summarized by stating that the proximal cause of the missing-letter effect is the time spent processing the target word. According to this view, readers process text in parallel at several levels of analysis, including feature, letter, syllable, and word levels. It is assumed that as soon as processing at a higher level is completed, processing at all lower levels is interrupted even if it was not completed. The failure to fully process a target letter within a word would translate into an omission. In this view, word function and word frequency would be distally related to omission rate by influ- 
encing word identification speed (see Saint-Aubin et al., 2003): There would be more omissions for high- than for low-frequency words because, due to their familiarity, the former are processed faster than the latter. In addition to word frequency, word function influences omission rate: There are more omissions for function words than for content words. According to the GO model, function words are processed faster than content words because readers use them to rapidly establish a tentative structural frame of the phrase or the sentence. Finally, within the GO model, eye movements contribute to omission rate because they influence the quality of the visual input. Omission rates would be higher for skipped than for fixated words (RoyCharland, Saint-Aubin, Klein, \& Lawrence, 2007; SaintAubin \& Klein, 2001) and for words with shorter gaze durations.

The GO model can successfully account for most of the database related to the missing-letter effect (Greenberg et al., 2004). However, it is unclear whether or not the GO model can account for the influence of text repetition, or rereading, because the influence of text repetition on the missing-letter effect is far from clear. Broadly put, the text repetition effect usually refers to an increase in the speed of reading or accuracy at related tasks caused by previous experience with the same or related reading materials (see, e.g., Raney, 2003). In the field of reading, rereading has been an important topic. There are multiple reasons for the interest in rereading. Among them, it is worth mentioning that rereading is a common and suitable strategy for learning new information, allows investigation of the influence of implicit memory on reading, and can be used to manipulate text difficulty (see, e.g., Greenberg \& Tai, 2001; Raney, 2003; Rayner, Raney, \& Pollatsek, 1995). For investigating the missing-letter effect, it is useful because it is a way of experimentally increasing word-processing speed while maintaining all other factors constant.

In multiple-readings studies, it is widely acknowledged that overall reading speed increases when participants are reading exactly the same text more than once (see, e.g., Levy, 1983; Levy, Di Persio, \& Hollingshead, 1992). Most importantly in the context of the missing-letter effect, when a text is read up to three times, gaze durations for high- and low-frequency words are reduced equivalently across reading (Raney \& Rayner, 1995; Rayner et al., 1995), but skipping increases for low- but not for high-frequency words (Raney \& Rayner, 1995). To the best of our knowledge, no study has separately assessed the effect of repetition on gaze durations as a function of word class. However, Raney and Rayner reported indirect evidence that led them to conclude that "some words, most likely function words, show little or no repetition benefits" (p. 164). According to the GO model, this pattern of eye movements should translate into a higher omission rate for content words when the text is reread than when it is read for the first time. The repetition effect on omission rate is also likely to be greater for low- than for high-frequency words. For function words, the influence of repetition should at best be very small, because eye movement data suggest that they are largely unaffected by repetition.
The predictions derived from the GO model are clear, whereas the influence of repetition on the missing-letter effect is far less so. One problem arises from the dual-task procedure of searching for a target letter while reading, which is necessary for observing the missing-letter effect. Consequently, the influences of multiple readings and of multiple searches usually covary, but it is necessary to isolate them from one other in investigating the influence of rereading on the missing-letter effect. Greenberg and Tai (2001) are the only ones to investigate the effect of text familiarity without the confounding influence of multiple searches (the study of Healy et al., 1987, is an example of a study in which repeated reading and repeated searching are confounded). In one experiment, Greenberg and Tai contrasted omission rates for target letters embedded in function and content words from well-known nursery rhymes (e.g., "Jack and Jill went up the hill to fetch a pail of water") with those for target letters embedded in words from unfamiliar, control sentences derived from the same nursery rhymes (e.g., "Amy and John went by the lake to catch a fish for later"). They found fewer omissions when the target letter $t$ was embedded in function words that were part of the familiar nursery rhymes than when it was embedded in function words of the control sentences. The reverse pattern was observed for content words. Although interesting, this experiment does not constitute a satisfactory test of the influence of multiple readings on the missing-letter effect. In effect, in Greenberg and Tai's experiment the text was read only once, and, as their name implies, nursery rhymes are usually learned long before children learn to read and are not usually read afterward. Thus, this manipulation is different from the rereading manipulation. Also, it is well established that omission rates can vary from one sentence to another despite the fact that the sentences were written to be as similar as possible (see, e.g., Saint-Aubin \& Klein, 2004).

The other experiment of Greenberg and Tai (2001) circumvented the above-mentioned problems by using a different methodology. Participants read a sentence aloud either two or five times and then were asked to read a short paragraph composed of four sentences. In these paragraphs, the repeated sentence was always reproduced. Furthermore, in half of the paragraphs there was also a control sentence containing some of the same test words. Results revealed a small decrease in omission rate for function words and a small increment for content words, resulting in a smaller missing-letter effect. However, once more, there are doubts concerning their results, this time because sentences were not counterbalanced across participants for the repeated and the nonrepeated conditions. With different sentences used in the single-reading and multiplereadings conditions, the effect observed by Greenberg and Tai could be due to sentence differences rather than to repetition. We tested this possibility by running an experiment with Greenberg and Tai's materials. Although in our experiment all sentences were presented only once, we reproduced their lower omission rate for the target letter $t$ in the when it was embedded in their repeated sentences rather than in their nonrepeated sentences. ${ }^{1}$ In addition, we ran another experiment with 24 participants. We used 
exactly the same methodology as Greenberg and Tai used, but in our case repeated and nonrepeated sentences were completely counterbalanced across participants. There was absolutely no repetition effect. In a nutshell, the procedure used by Greenberg and Tai was not the most appropriate for uncovering a repetition effect, because in the multiple-readings condition the earlier readings were not performed in the same conditions as the final reading and there was not enough content to allow the emergence of a repetition effect (see, e.g., Levy, 2001; Raney, 2003).

The main purpose of the experiments reported here was to implement the first comprehensive investigation of the influence of multiple readings on the missing-letter effect with an adequate methodology. In Experiment 1, participants either read the same text once or read it three times and performed the letter search task on the third reading. In the latter case, they were either aware or unaware, from the beginning of the experiment, of the target letter to be used on the third reading. In Experiments 2 and 3, in the multiple-readings condition, participants searched for a different target letter on each reading to promote the establishment of similar situation models across reading without the confounding effect of multiple searches of the same target letter.

\section{EXPERIMENT 1}

To date, the influence of multiple readings when readers are also searching for the target letter on each reading has been investigated only once, in a study not specifically designed to address this issue (Healy et al., 1987). Nevertheless, the results revealed that, from the first to the third reading, the omission rate decreased from $23.6 \%$ to $6.9 \%$ for the correctly spelled function word the and from $7.4 \%$ to $2.1 \%$ for correctly spelled content words. However, it is not clear whether this effect is due to multiple searches, to multiple readings, or to both. In addition, the procedure used by Healy et al. was not the most appropriate, and their major findings were subsequently invalidated (Saint-Aubin $\&$ Klein, 2001). In the present experiment, the influences of multiple readings and multiple searches are isolated. The baseline is provided by a group of participants (the single-reading group) reading the text once while searching for the target letter. The influence of multiple readings with multiple searches was investigated by having a group of participants who were aware, from the start of the first reading, of the target letter to be used on the last reading (the aware multiple-readings group) read the text three times and perform the search task on the third reading only. This procedure, with an implicit search on the initial readings, was preferred over an explicit search of the target letter on each reading because it has been shown that letter search slows down reading, which could prevent reading speed from increasing (Greenberg, Inhoff, \& Weger, 2006; Oliver, Healy, \& Mross, 2005; Roy-Charland et al., 2007). Finally, the influence of multiple readings was isolated by having a group of participants (the unaware multiplereadings group) read the text three times and perform the search task on the third reading only, without being aware in advance of the target letter to be used on the final reading. For both multiple-readings groups, the number of readings was fixed at three to allow direct comparison with previous studies. In effect, as seen above, eye movement studies provide data on up to three readings, which is also the smallest number of readings in the repetition condition of the only previous study to investigate the impact of repetition on the missing-letter effect (Greenberg \& Tai, 2001).

\section{Method}

Participants. A total of 72 unpaid undergraduate students (48 females, 24 males) took part in the experiment, with 24 participants per reading group. All participants had French as their first language.

Materials and Procedure. The des text used by Saint-Aubin and Klein (2004; Saint-Aubin et al., 2003) was used here. The text comprised 593 words and contained 24 instances of the French plural indefinite article des, with a frequency count of 15,877 occurrences per million, and 24 instances of three-letter control content words beginning with the target letter $d$, with an average frequency count of 248 occurrences per million (e.g., don [donation], dit [says], dix [ten], dos [back], duo [duet]) (New, Pallier, Ferrand, \& Matos, 2001). In addition, the target letter $d$ was embedded in 14 noncritical words. Each word containing the target letter, be it critical or not, was separated from the previous and the following words by at least 4 filler words not containing the target letter. The test words were not included in the first and last sentences of the text, were never at the beginning or the end of a sentence or a line, and were never adjacent to a punctuation mark.

A stapled booklet was assembled. On the first sheet, the instructions were presented. For the multiple-readings conditions, the instructions stated that participants were required to read a one-page text three times. They were informed that the text was printed three times on three different pages. Each text page was followed by a page with three comprehension questions. Three sets of comprehension questions were created to promote reading for comprehension. The first set was administered to all participants in the single-reading condition. For both multiple-readings groups, the first set was always administered first, followed by the second set on the second reading and the third set on the third reading. These participants were informed that they should read the text on all occasions for meaning, and that for the first two readings they would be reading only and should do so aloud. It was also mentioned that for the third reading they would read silently but also perform a letter search task. The participants in the aware multiple-readings group were informed that the target letter on the third reading would be the letter $d$ and that this letter was reproduced at the top of the three text pages. Participants in the unaware multiple-readings group were informed that the target letter would be indicated before the third reading. All participants were reminded that, the search task notwithstanding, they should read the text for comprehension at their normal reading speed. They were told that whenever they came across the target letter (whether in uppercase or in lowercase), they were to circle it. They were warned not to slow down their reading to detect all target letters and not to backtrack to circle a target letter they had missed. To familiarize participants with the search task, they were asked to read the instruction sheet again and to circle all $t$ s. Finally, an additional instruction page was inserted between the page with the comprehension questions for the second reading and the page with the third presentation of the text. Those instructions reminded participants that for the next reading they would also perform the letter search task. Then the participants in the aware multiple-readings group were simply reminded that the target letter was $d$, whereas the unaware participants were informed for the first time that the target letter was $d$. Participants were reminded to read normally and for comprehension. For the participants in the single-reading condition, the first part of the instructions about the first two readings was omitted. 
Testing was done individually in one session lasting approximately $15 \mathrm{~min}$ for those in the multiple-readings conditions and approximately $10 \mathrm{~min}$ for those in the single-reading condition. Participants were assigned to one of the three reading groups at random, with the constraint that there must be 24 participants per group. The experimenter was present throughout the experiment to ensure compliance with the instructions.

\section{Results}

Comprehension questions were analyzed first. Overall, participants were highly accurate in answering the questions, with an average success rate of $94 \%$ or higher in all but the single-reading condition. More specifically, results revealed that, on average, participants in the single-reading group correctly answered the comprehension questions $85 \%$ of the time, whereas those in the unaware and aware multiple-readings groups answered correctly $94 \%$ and $99 \%$ of the time, respectively, on the first reading. The lower score observed for the single-reading group is probably due to the fact that, unlike participants in the other two groups, those in this group were reading and overtly detecting the letters. This overt detection behavior might have hindered readers' memory for story details by forcing them to divide their attention between the two tasks. The between-subjects ANOVA on the first reading with reading group as factor was significant $[F(2,69)=4.66$, $\left.M S_{\mathrm{e}}=261.67\right]$. Post hoc comparisons (Student/NewmanKeuls) revealed that participants in the single-reading group were significantly worse at answering the comprehension questions than those in the two multiple-readings groups, which did not differ from each other.

Mean percentages of omission errors for des and for the control content words are presented in the top panel of Figure 1 as a function of reading condition. Inspection of this figure reveals that readers in all three groups made more omissions of the target letter $d$ when it was embedded in the frequent function word des than when it was embedded in the less frequent content words. There were also fewer omissions for the function word des when participants read the text three times and knew from the first reading which letter they would be looking for in the third reading, in comparison with when the text was read once or when it was read three times without advance knowledge of the target letter's identity. For content words, there was no obvious difference between the three reading conditions. Consequently, a smaller missing-letter effect — as measured by subtracting omissions for the content words from omissions for the frequent function word des-was observed for readers in the aware multiple-readings group than for those in the other two conditions.

A $3 \times 2$ mixed-design ANOVA with reading condition as the between-subjects factor and word function as the repeated factor revealed significant main effects of word function $\left[F(1,69)=36.02, M S_{\mathrm{e}}=172.49\right]$ and of the interaction between word function and reading condition $\left[F(2,69)=3.53, M S_{\mathrm{e}}=172.49\right]$. The main effect of reading condition was not significant $(F \leq 1)$. Simple main effect tests revealed that the effect of word function was significant for the single-reading group $[F(1,69)=12.43$, $\left.M S_{\mathrm{e}}=172.49\right]$ and for the unaware multiple-readings
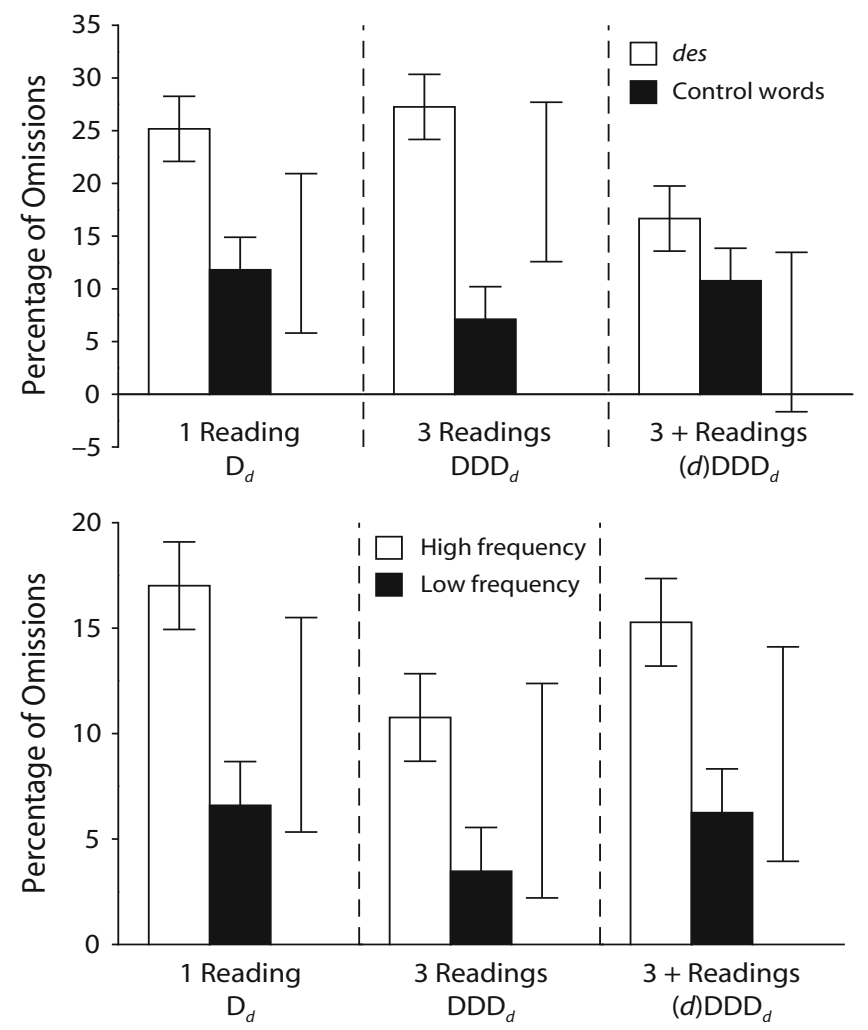

Figure 1. Mean percentage of omissions in Experiment 1 as a function of word function and reading condition (top panel) and word frequency among content words and reading condition (bottom panel). The three reading conditions are represented by both the numbers $(1,3$, and $3+$ advanced letter knowledge) and the letter code. Each uppercase $D$ represents one reading of the text. The subscript lowercase $d$ represents the letter used for the search task. The absence of a subscript letter means that there was no letter detection task on that reading, and the letter in parentheses indicates that the target letter identity was disclosed on that reading even though there was no detection requirement. Error bars on omission rates represent $95 \%$ confidence intervals for the within-subjects factor of word function or word frequency, computed according to the method of Loftus and Masson (1994). The error bars beside the bars of each condition are centered on the mean of the missing-letter size (top panel, omission rate on des minus omission rate on content words; bottom panel, omission rate on high-frequency words minus omission rate on lowfrequency words). Those error bars represent $95 \%$ confidence intervals for the between-subjects effect of repetition. When the interaction between word function or word class and repetition is significant across repetition conditions, those confidence intervals overlap by no more than about half the distance of one side of an interval (see Masson \& Loftus, 2003).

group $\left[F(1,69)=28.90, M S_{\mathrm{e}}=172.49\right]$, but not for the aware multiple-readings group $(F=2.42)$.

Another series of analyses was conducted on the 24 instances of the critical content words. These analyses are aimed at investigating the effect of reading condition on the missing-letter effect as obtained by the contrast between high- and low-frequency words. For these analyses, the content words with fewer than 100 occurrences per million were assigned to the low-frequency category, and those with greater than 100 occurrences per million were 
assigned to the high-frequency category. With these criteria, 12 occurrences of the critical content words were assigned to the low-frequency category ( $M=41$ occurrences per million) and 12 were assigned to the highfrequency category ( $M=411$ occurrences per million).

Mean percentages of omissions as a function of frequency and reading condition are presented in the bottom panel of Figure 1. Inspection of this figure reveals a higher omission rate for the high- than for the lowfrequency content words for all three reading conditions. A $3 \times 2$ mixed-design ANOVA with reading condition as the between-subjects factor and word frequency as the repeated factor revealed a significant main effect of word frequency $\left[F(1,69)=36.67, M S_{\mathrm{e}}=77.98\right]$, but neither the main effect of repetition $(F=1.13)$ nor the interaction $(F \leq 1)$ was significant.

\section{Discussion}

The results of Experiment 1 reveal a clear missingletter effect for all three reading conditions when omission rates for high- and low-frequency content words are contrasted. However, when the frequent function word des was contrasted with less frequent content words, the usual missing-letter effect was observed in all but the aware multiple-readings group, in which the target letter was disclosed before the first reading. Results for the aware multiple-readings group fit well with the results of Healy et al. (1987), in which participants performed a letter search task with the same target letter on each reading. There were fewer omissions on the third than on the first reading, especially for the high-frequency function word the. As would be expected, the present results reflect that, even though our readers were not instructed to perform the letter search task on the first two readings, they were doing so and were improving just as did the participants in Healy et al.'s study, who were instructed to do so. The fact that the advantage was restricted to the high-frequency function word des might simply reflect the fact that it is easier to keep track of readings of the already detected target letter in a word repeated 24 times within the text than in a series of different words. In other words, for a repeated high-frequency function word, such as the in Healy et al.'s study or des in the present study, participants could use both the target letter and the critical word identity as retrieval cues, which would increase the likelihood of detecting the target letter on those words on the third reading.

The failure to find a repetition effect in the unaware multiple-readings group could be seen as a demonstration that the missing-letter effect is immune to multiple readings per se. Alternatively, it could be argued that multiple readings can influence the missing-letter effect, but only under some conditions that were not present in Experiment 1 . This possibility is addressed in Experiment 2.

\section{EXPERIMENT 2}

According to the context-dependent representation model, the more similar the situation models of successive readings, the larger the text repetition effects will be
(Raney, 2003). It could be argued that in Experiment 1 two elements reduce the similarity of the situation models. First, although in Experiment 1 participants were reading for comprehension on all readings, as in Greenberg and Tai's (2001) study they read aloud for the first two readings and silently for the final reading, during which the search task was performed. It could be argued that the change from reading aloud to reading silently creates two different situation models. In addition, in the missingletter effect literature, it is usually assumed that the search task is performed in parallel to the reading task. However, it could easily be argued that the search task is part of the situation model associated to a specific reading. Consequently, even if participants were reading silently on each occasion, the introduction of the search task on the last reading would create a new and distinct situation model, preventing the observation of an effect of repetition on the missing-letter effect.

This possibility was tested in Experiment 2. Accordingly, participants read the text used in Experiment 1 three times silently for comprehension. Participants performed a search task for a different letter on each reading. The use of a different letter on each reading was necessary to unconfound the impact of incorporating a letter search task into the situation models from the impact of searching many times for the same target letter. In addition, to remove the ceiling effect found for comprehension in the repetition groups of Experiment 1, more comprehension questions were asked after each reading, they were more difficult, and they were counterbalanced. Comprehension questions served as an independent index of the influence of repetition.

\section{Method}

Participants. A total of 102 students in Grade 12 (47 females, 53 males, 2 unspecified) from a French public school in New Brunswick took part in the experiment. All the participants had French as their first language. The participants were tested in June, which is very close to the end of the school year, and they were on average 17.6 years old (range, 17-20 years old). No participant had taken part in Experiment 1.

Materials and Procedure. The text used in Experiment 1 was used here. In addition, three sets of five comprehension questions were created. The comprehension questions were more difficult than those used in Experiment 1. The questions were aimed at testing the participants' memory for the details of the story. In the singlereading condition, about one third of the participants received each series of questions, with 16 participants answering questions of the first set, 17 answering questions of the second set, and 16 answering questions of the third set. For the repeated condition, the presentation order of the comprehension questions was counterbalanced across readings, with each of the six possible presentation orders of the question sets being given to $8-10$ participants.

The procedure was the same as in Experiment 1 except for the following modifications. There were only two conditions: single reading and multiple readings. Participants were tested in groups in their usual classrooms. A total of four classes participated. For the repetition condition, participants were instructed to read the text silently for comprehension. On each reading, a different target letter was assigned. The target letter $m$ (embedded in 65 words) was always used for the first reading, the target letter $f$ (embedded in 37 words) was used for the second reading, and, obviously, the target letter $d$ (embedded in 62 words, as in Experiment 1) was used for the third reading. For the nonrepetition condition, after reading the critical text, participants read an additional text and performed the 

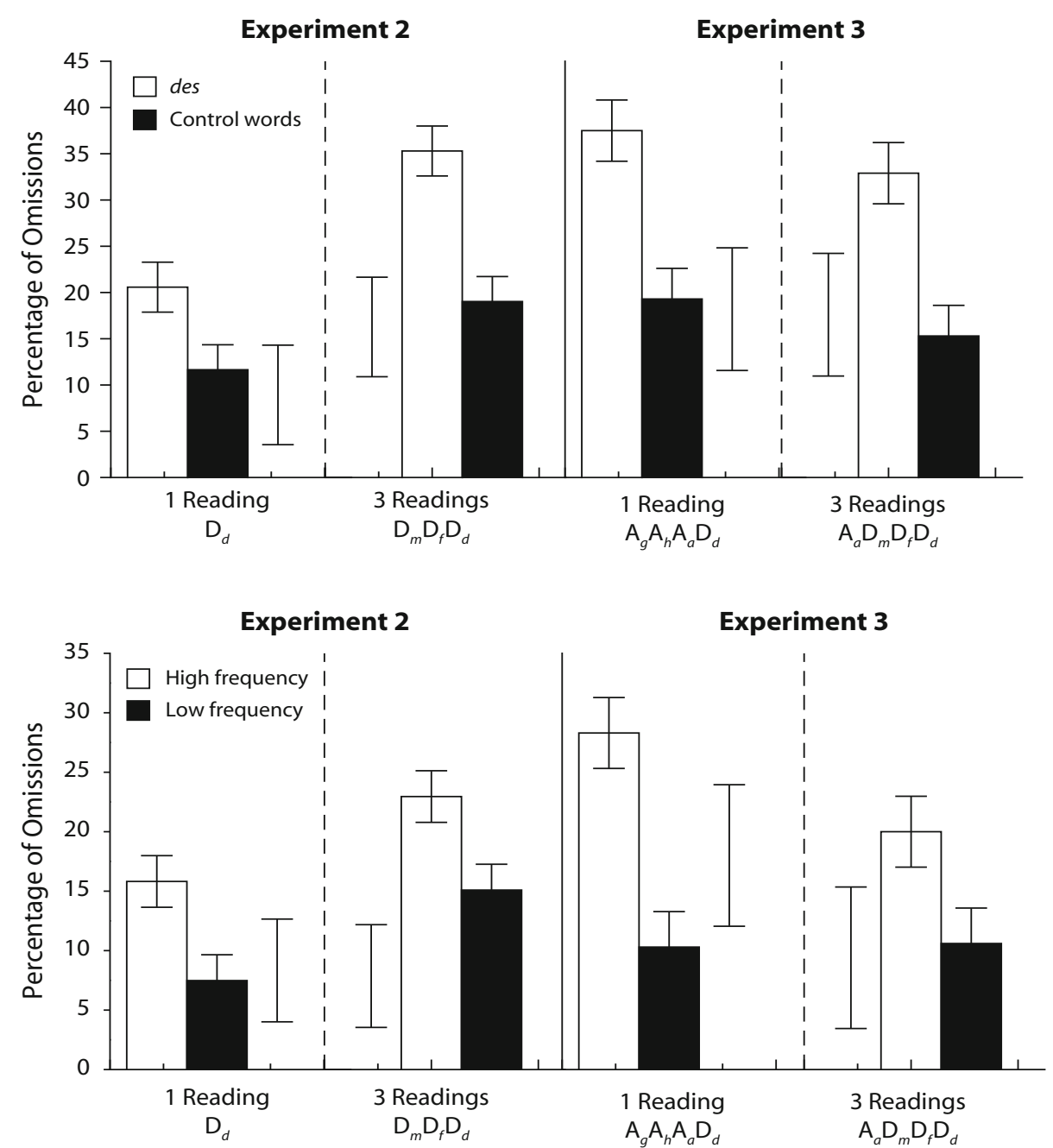

Figure 2. Mean percentage of omissions in Experiment 2 (left panels) and Experiment 3 (right panels) as a function of word function and reading condition (top panel) and word frequency of content words and reading condition (bottom panel). The error bars represent omission rates for the target letter $d$. Reading conditions are represented by the letter code as in Figure 1. Different uppercase letters represent different texts read, and each subscript letter represents the letter used for the search task on that reading. See Figure 1 for a thorough explanation of the error bars.

letter search task on this text. The additional text was included only to provide an experience of the same length for all participants and to make sure that the experiment was at first glance identical for all participants, and it was not analyzed.

\section{Results}

Performance on comprehension questions was analyzed first. Results revealed that, on average, participants in the single-reading group correctly answered the five comprehension questions $70 \%$ of the time, and those in the multiple-readings group did so $72 \%$ of the time after the first reading. This difference was not significant $(F \leq 1)$. For the multiple-readings group, the score on the comprehension questions increased to $76 \%$ after the second reading and to $82 \%$ after the third reading. A repeated measures ANOVA revealed a significant effect of number of readings $\left[F(2,104)=3.68, M S_{\mathrm{e}}=347.22\right]$. Post hoc comparisons (Student/Newman-Keuls) revealed that participants were significantly better at answering the comprehension questions after the third reading than after the first. The second reading did not differ from the first or the third reading.

Mean percentages of omission errors for des and for the control content words are presented in the top panel of Figure 2 as a function of repetition. Inspection of this figure reveals that, for both the single-reading and the multiple-readings conditions, readers made more omissions of the target letter $d$ when it was embedded in the frequent function word des than when it formed part of the less frequent content words. Omission rate increased for both des and the content words when the text was repeated, but the increase was more pronounced for des. 
A $2 \times 2$ mixed-design ANOVA with repetition as the between-subjects factor and word function as the repeated factor revealed significant main effects of word function $\left[F(1,99)=42.91, M S_{\mathrm{e}}=187.12\right]$ and repetition $[F(1,99)=$ $\left.10.94, M S_{\mathrm{e}}=568.44\right]$ and a marginally significant interaction between word function and repetition $[F(1,99)=$ 3.68, $\left.M S_{\mathrm{e}}=187.12, p=.058\right]$. Simple main effect tests revealed that the effect of word function was significant for the single-reading condition $\left[F(1,99)=10.26, M S_{\mathrm{e}}=\right.$ $187.12]$ and the three-readings condition $[F(1,99)=$ $\left.37.51, M S_{\mathrm{e}}=187.12\right]$. In addition, the effect of repetition was significant for des $\left[F(1,200)=14.55, M S_{\mathrm{e}}=379.08\right]$ and marginally significant for the control content words $\left[F(1,200)=3.65, M S_{\mathrm{e}}=379.08, p=.057\right]$.

Another series of analyses was conducted on the 24 instances of the critical content words. As is shown in the bottom panel of Figure 2, there were more omissions for the high- than for the low-frequency content words both when the text was read once and when it was read repeatedly. The omission rate also increased by similar amounts for the high- and the low-frequency words on the third reading in comparison with the first reading. A $2 \times 2$ mixed-design ANOVA with repetition as the between-subjects factor and word frequency as the repeated factor revealed significant main effects of word frequency $\left[F(1,99)=28.05, M S_{\mathrm{e}}=120.94\right]$ and repetition $\left[F(1,99)=9.11, M S_{\mathrm{e}}=304.07\right]$, but the interaction was not significant $(F \leq 1)$.

\section{EXPERIMENT 3}

Although the results of Experiment 2 are clear and interesting, they warrant replication because they are the first demonstration of the influence of multiple readings on the missing-letter effect. In addition, it could be argued that there was a confounding factor in Experiment 2. In effect, in the repetition condition, the higher omission rate on the third reading than on the first could be due to faster word processing or to the amount of previous letter searching. It might be argued, for example, that searching for a target letter on three consecutive readings reduces either the vigilance or the motivation of the reader. One way of circumventing this potential problem is to ask readers in the single-reading condition to first read three times an irrelevant text of exactly the same length but on a different topic and to search on each reading for a different target letter. Following these three initial readings, participants would read the critical text and search for the target letter. In the multiple-readings condition, participants would read the irrelevant text once while searching for a target letter and then read the critical text three times, searching for a different target letter on each reading. This procedure ensures that, in both the single-reading and the multiple-readings conditions, the fourth reading is the critical reading and all participants read two different texts. Under these conditions, it would not be possible to attribute a higher omission rate on the third reading than on the first to the amount of previous letter search. In addition, contrary to Experiment 1, if there is no repetition effect on omission rate, this could not be attributed to the fact that the situation model of the first two readings of the critical text did not match the situation model of the final reading, because on all readings participants were also searching for a target letter.

\section{Method}

Participants. A total of 60 undergraduate students ( 31 females, 25 males, 4 unspecified) took part in the experiment. All had French as their first language, and none of them had taken part in either of the previous experiments.

Materials and Procedure. The text and the comprehension questions used in Experiment 2 were used here. In the single-reading condition, about one third of the participants received each series of questions, with 9 participants answering questions of the first set, 11 answering questions of the second set, and 10 answering questions of the third set. For the repeated condition, the presentation order of the comprehension questions was counterbalanced with a Latin square design, with 8,11 , and 11 participants each receiving one of the three possible orders.

The procedure was the same as in Experiment 2 except for the following modifications. All participants first read an unrelated text comprising 593 words, which is exactly the same length as the critical text. After each reading, they answered a series of five multiple-choice questions. In the single-reading condition, participants read the unrelated text three times and searched for the target letter $g$ (embedded in 59 words) on the first reading, the target letter $h$ (embedded in 45 words) on the second reading, and the target letter $a$ (embedded in 52 words) on the third reading. The critical text was then presented, and they searched for the target letter $d$. In the multiple-readings condition, participants read the unrelated text once and searched for the target letter $a$. They then read the critical text three times and, as in Experiment 2, searched for the target letters $m, f$, and $d$ on the first, second, and third readings, respectively.

\section{Results}

Performance on comprehension questions was analyzed first. Results revealed that, on average, participants in the single-reading group correctly answered the five comprehension questions $64 \%$ of the time, and those in the multiple-readings group did so $73 \%$ of the time after the first reading. This difference was not significant $(F=$ 2.64). For the multiple-readings group, the score on the comprehension questions increased to $79 \%$ after the second reading and to $80 \%$ after the third reading. The differences did not reach significance $(F=1.81)$.

Mean percentages of omission errors for des and for the control content words as a function of repetition are presented in the top panel of Figure 2. Inspection of this figure reveals that, for both the single-reading and the multiple-readings conditions, readers made more omissions of the target letter $d$ when it was embedded in the frequent function word des than when it was embedded in the less frequent content words. Participants in the repetition group made slightly fewer omissions on both critical words than those in the nonrepetition group. The $2 \times 2$ mixed-design ANOVA with repetition as the between-subjects factor and word function as the repeated factor revealed a significant main effect of word function $\left[F(1,58)=58.58, M S_{\mathrm{e}}=164.38\right]$, but neither the main effect of repetition nor the interaction reached significance (both $F_{\mathrm{s}} \leq 1$ ).

Another series of analyses was conducted on the 24 instances of the critical content words. Inspection of the 
bottom panel of Figure 2 reveals a higher omission rate for the high- than for the low-frequency content words both when the text was read once and when it was read repeatedly. In addition, participants in the repetition group made fewer omissions on the frequent content words than did those in the single-reading group. The ANOVA revealed significant effects of word frequency $[F(1,58)=42.75$, $\left.M S_{\mathrm{e}}=132.68\right]$ and of the interaction $[F(1,58)=4.19$, $\left.M S_{\mathrm{e}}=132.68\right]$, but the main effect of repetition did not reach significance $(F=1.21)$. Simple main effect tests revealed that there were significantly more omissions when the target letter was embedded in high- than in lowfrequency words for both the single-reading $[F(1,58)=$ $\left.36.86, M S_{\mathrm{e}}=132.68\right]$ and the multiple-readings $\left[F(1,58)=10.08, M S_{\mathrm{e}}=132.68\right]$ groups. In addition, the effect of repetition was significant for high-frequency words $\left[F(1,116)=3.90, M S_{\mathrm{e}}=266.78\right]$ but not for lowfrequency words $(F \leq 1)$.

Over and above the specific results of Experiments 2 and 3 , the most striking effect depicted in Figure 2 is indisputably the similarity of performance on the third reading of the critical text in Experiment 2 (condition $\mathrm{D}_{m} \mathrm{D}_{f} \mathrm{D}_{d}$ ) and on the first reading of the critical text in Experiment 3, which is overall the fourth text read (condition $\mathrm{A}_{g} \mathrm{~A}_{h} \mathrm{~A}_{a} \mathrm{D}_{d}$ ). We investigated this effect by running an additional mixeddesign ANOVA on those two conditions, with experiment (condition $\mathrm{D}_{m} \mathrm{D}_{f} \mathrm{D}_{d}$ of Experiment 2 vs. condition $\mathrm{A}_{g} \mathrm{~A}_{h} \mathrm{~A}_{a} \mathrm{D}_{d}$ of Experiment 3 ) as the between-subjects factor and word function (des vs. control content words) as the repeated factor. Results revealed a significant main effect of word function $\left[F(1,81)=55.84, M S_{\mathrm{e}}=204.42\right]$, but neither the main effect of experiment nor the interaction reached significance (both $F \mathrm{~s} \leq 1$ ). A similar analysis was performed for word frequency (see the bottom panel of Figure 2). The ANOVA revealed significant effects of word frequency $\left[F(1,81)=59.70, M S_{\mathrm{e}}=107.59\right]$ and of the interaction $\left[F(1,81)=9.25, M S_{\mathrm{e}}=107.59\right]$, but the main effect of repetition did not reach significance $(F \leq 1)$. On the one hand, simple main effect tests revealed that there were significantly more omissions when the target letter was embedded in high- than in low-frequency words both for condition $\mathrm{D}_{m} \mathrm{D}_{f} \mathrm{D}_{d}$ of Experiment $2[F(1,81)=$ 15.22, $\left.M S_{\mathrm{e}}=107.59\right]$ and for condition $\mathrm{A}_{g} \mathrm{~A}_{h} \mathrm{~A}_{a} \mathrm{D}_{d}$ of Experiment $3\left[F(1,81)=45.45, M S_{\mathrm{e}}=107.59\right]$. On the other hand, simple main effect tests revealed that the effect of experiment was significant neither for rare $(F=2.25)$ nor for frequent $(F=1.80)$ words.

\section{Discussion}

In Experiment 3, the procedure was the same as in Experiment 2: The same text was used, and participants were required to search for a target letter on each reading. The only difference was that the influence of the amount of previous letter search was controlled for by the inclusion of an irrelevant text before the critical text was read. With the inclusion of the irrelevant text, both the third reading in the multiple-readings condition and the first reading in the single-reading condition were performed on the fourth text read. Even with this additional control, a substantial missing-letter effect was observed in both the single- reading and the multiple-readings conditions. However, the influence of repetition vanished completely when the omission rate for des was contrasted with that for control content words. When the content word data were separated by word frequency (Figure 2, bottom panel), there was a significant effect only for high-frequency words, with a smaller omission rate on the third reading than on the first reading.

Two important findings emerged from this experiment. First, the missing-letter effect was completely unaffected by familiarity with a text when the amount and variety of letter search prior to the critical text was controlled. Second, the global increase in omissions seen in Experiment 2 , which could have been due to familiarity with the text or to the amount of prior letter searching, is demonstrated to be due entirely to the latter factor. This conclusion is further supported by the fact that performance on the single-reading condition in Experiment 3 was indistinguishable from performance on the third reading in Experiment 2. In both cases, the amounts of previous letter search were similar, but levels of familiarity with the critical text were very different.

\section{GENERAL DISCUSSION}

The present study offers the first comprehensive examination of the impact of multiple readings on the missingletter effect. Results revealed that when participants were aware of the target letter's identity from the start, there were fewer omissions on the third than on the first reading for the repeated high-frequency function word des. These results are very compatible with Healy et al.'s (1987) results, which indicate that the omission rate decreased mostly for the correctly spelled function word the when readers were searching for the same target letter on each reading. Our results can be interpreted by assuming that, even when they were not instructed to do so, readers were searching for the target letter on each reading. This could be considered an indication of the influence of multiple searches on the missingletter effect. With regard to the influence of multiple readings, results of our Experiment 1 indicate that if the letter search is not added until the third reading, there is no effect of multiple readings. Results of Experiment 2 suggest that the search task must be performed on each reading in order for a repetition effect to be observed. However, results of Experiment 3 revealed that the effects observed in Experiment 2 were due to the amount of prior letter search. In effect, when the amount of previous reading and the amount of searching are equated between the single-reading and the multiple-readings conditions, the repetition effect almost completely vanishes. In sum, the overall conclusion seems to be that the missing-letter effect is simply unaffected by repetition.

It is worth noting that the present results are very similar to those of Levy et al. (1992), who investigated the impact of multiple readings on detection accuracy for misspellings. Although there are substantial differences between detection of misspellings and letter detection in correctly spelled words (see Healy, 1994, for a review), the patterns of results observed here and by Levy et al. are strikingly 
similar. More precisely, in one set of experiments, Levy et al. had readers detect spelling errors across multiple readings. Results revealed that with repetition, readers read faster but error detection rates remained constant or slightly improved. This pattern of results was observed despite the fact that different misspellings were introduced on each reading, a strategy that is similar to our use of different target letters. Thus, just as in the present study, increased processing speed did not reduce readers' ability to detect the target. In sum, Levy et al.'s study provides converging evidence that multiple readings do not influence detection accuracy at the letter level.

In light of the present results, where do we stand? Greenberg and Tai's (2001) study provides, in part, the impetus for the present study. Greenberg and Tai observed that there were fewer omissions for function words in the multiple-reading condition than in the single-reading condition, but the pattern was exactly the opposite for content words. These opposing trends were expected to reduce the size or abolish the missing-letter effect in the multiplereadings condition. As we mentioned in the introduction, Greenberg and Tai's results were based on a less-thanoptimal experimental design. In light of the present results, it seems legitimate to suggest that Greenberg and Tai's conclusions are unwarranted.

Now that the influence of repetition on the missingletter effect has been clarified, it remains to be seen how it fits within the GO model (Greenberg et al., 2004). According to the GO model, whenever a word is processed faster, omission rate increases. As previous eye movement studies have shown, processing speed increases over repetition, and more so for content than for function words and for rare than for frequent content words. Consequently, the GO model predicts more omissions on the third than on the first reading, and a smaller missing-letter effect, because the increase would be more pronounced for content than for function words and for low- than for high-frequency words. The present results show no increment of omission rate across readings, and the only significant effect in Experiment 3 was a lower omission rate for high-frequency words on the third reading than on the first reading. In sum, modifications should be introduced to the GO model to enable it to account for repetition effects. In light of the present results, if proponents of the GO model want to keep word identification speed as the major determinant of omission rate, they will have to drop the independence hypothesis. In effect, the GO model assumes that letterprocessing speed is unaffected by word-processing speed. In order to account for the present results, it would have to be assumed that, under some circumstances, such as repetition, word identification and letter identification increase at the same rate. This would account nicely for present results, but it would require the inclusion of a rule for determining under which circumstances word- and letter-processing speed are independent and under which circumstances they are not.

In conclusion, the present study offers the first comprehensive examination of the impact of multiple readings on the missing-letter effect. Results clearly indicate that (1) the missing-letter effect is completely unaffected by familiarity with a text, (2) multiple searches of the same target letter in the same text can be beneficial to the detection of this target letter when it is embedded in a frequently repeated high-frequency function word, and (3) detection accuracy decreases as the amount of previous letter search increases, be it on the critical text or elsewhere.

\section{AUTHOR NOTE}

This research was supported by discovery grants from the Natural Sciences and Engineering Research Council of Canada to J.S.-A. and to R.M.K. We thank Seth Greenberg for making his testing materials available to us. We also thank Hilary D. Duncan for her helpful comments on an earlier version of this article, and the Cité-des-Jeunes A. M. Sormany school for its collaboration in data collection. Correspondence concerning this article should be addressed to J. Saint-Aubin, École de Psychologie, Université de Moncton, Moncton, NB, E1A 3E9 Canada (e-mail: jean.saint-aubin@umoncton.ca).

\section{REFERENCES}

Assink, E. M. H., \& KNuiJt, P. P. N. A. (2000). Reading development and attention to letters in words. Contemporary Educational Psychology, 25, 347-362.

Assink, E. M. H., van Well, S., \& KnuiJt, P. P. N. A. (2003). Contrasting effects of age of acquisition in lexical decision and letter detection. American Journal of Psychology, 116, 367-387.

Corcoran, D. W. J. (1966). An acoustic factor in letter cancellation. Nature, 210, 658.

Cunningham, T. F., Healy, A. F., Kanengiser, N., Chizzick, L., \& WillitTs, R. L. (1988). Investigating the boundaries of reading units across ages and reading levels. Journal of Experimental Child Psychology, 45, 175-208.

DREWNOWSKI, A. (1978). Detection errors on the word the: Evidence for the acquisition of reading levels. Memory \& Cognition, 6, 403-409.

Drewnowski, A. (1981). Missing -ing in reading: Developmental changes in reading units. Journal of Experimental Child Psychology, 31, 154-168.

Drewnowski, A., \& Healy, A. F. (1977). Detection errors on the and and: Evidence for reading units larger than the word. Memory \& Cognition, 5, 636-647.

Greenberg, S. N., Healy, A. F., Koriat, A., \& Kreiner, H. (2004). The GO model: A reconsideration of the role of structural units in guiding and organizing text on line. Psychonomic Bulletin \& Review, 11, 428-433.

Greenberg, S. N., Inhoff, A. W., \& Weger, U. W. (2006). The impact of letter detection on eye movement patterns during reading: Reconsidering lexical analysis in connected text as a function of task. Quarterly Journal of Experimental Psychology, 59, 987-995.

GreenberG, S. N., \& TAI, J. (2001). Letter detection in very familiar texts. Memory \& Cognition, 29, 1088-1095.

Healy, A. F. (1994). Letter detection: A window to unitization and other cognitive processes in reading text. Psychonomic Bulletin \& Review, 1, 333-344.

Healy, A. F., Oliver, W. L., \& McNamara, T. P. (1987). Detecting letters in continuous text: Effects of display size. Journal of Experimental Psychology: Human Perception \& Performance, 13, 279-290.

Koriat, A., \& GreenberG, S. N. (1991). Syntactic control of letter detection: Evidence from English and Hebrew nonwords. Journal of Experimental Psychology: Learning, Memory, \& Cognition, 17, 1035-1050.

Koriat, A., \& Greenberg, S. N. (1994). The extraction of phrase structure during reading: Evidence from letter detection errors. Psychonomic Bulletin \& Review, 1, 345-356.

Levy, B. A. (1983). Proofreading familiar text: Constraints on visual processing. Memory \& Cognition, 11, 1-12.

LEVy, B. A. (2001). Text processing: Memory representations mediate fluent reading. In M. Naveh-Benjamin, M. Moscovitch, \& H. L. Roediger III (Eds.), Perspectives on human memory and cognitive aging: Essays in honour of Fergus Craik (pp. 83-98). New York: Psychology Press.

Levy, B. A., Di Persio, R., \& Hollingshead, A. (1992). Fluent reread- 
ing: Repetition, automaticity, and discrepancy. Journal of Experimental Psychology: Learning, Memory, \& Cognition, 18, 957-971.

Loftus, G. R., \& Masson, M. E. J. (1994). Using confidence intervals in within-subject designs. Psychonomic Bulletin \& Review, 1, 476-490.

Masson, M. E. J., \& Loftus, G. R. (2003). Using confidence intervals for graphically based data interpretation. Canadian Journal of Experimental Psychology, 57, 203-220.

Mohan, P. J. (1978). Acoustic factors in letter cancellation: Developmental considerations. Developmental Psychology, 14, 117-118.

MoravcsiK, J. E., \& Healy, A. F. (1995). Effect of meaning on letter detection. Journal of Experimental Psychology: Learning, Memory, \& Cognition, 21, 82-95.

Oliver, W. L., Healy, A. F., \& Mross, E. F. (2005). Trade-offs in detecting letters and comprehending text. Canadian Journal of Experimental Psychology, 59, 159-167.

New, B., Pallier, C., Ferrand, L., \& Matos, R. (2001). Une base de données lexicales du français contemporain sur Internet: Lexique [A lexical database for contemporary French on the Internet: Lexique]. L'Année Psychologique, 101, 447-462.

Raney, G. E. (2003). A context-dependent representation model for explaining text repetition effects. Psychonomic Bulletin \& Review, 10, 15-28.

RANEY, G. E., \& RAYNER, K. (1995). Word frequency effects and eye movements during two readings of a text. Canadian Journal of Experimental Psychology, 49, 151-173.

Rayner, K., Raney, G. E., \& Pollatsek, A. (1995). Eye movements and discourse processing. In R. F. Lorch Jr., \& E. J. O’Brien (Eds.), Sources of coherence in reading (pp. 9-35). Hillsdale, NJ: Erlbaum.

READ, J. D. (1983). Detection of Fs in a single statement: The role of phonetic recoding. Memory \& Cognition, 11, 390-399.

Roy-Charland, A., \& Saint-Aubin, J. (2006). The interaction of word frequency and word class: A test of the GO model's account of the missing-letter effect. Quarterly Journal of Experimental Psychology, 59, 38-45.

Roy-Charland, A., Saint-Aubin, J., Klein, R. M., \& Lawrence, M. (2007). Eye movements as direct tests of the GO model for the missing-letter effect. Perception \& Psychophysics, 69, 324-337.

Saint-Aubin, J., \& Klein, R. M. (2001). Influence of parafoveal processing on the missing-letter effect. Journal of Experimental Psychology: Human Perception \& Performance, 27, 318-334.

Saint-Aubin, J., \& Klein, R. M. (2004). One missing-letter effect: Two methods of assessment. Canadian Journal of Experimental Psychology, 58, 61-66.

SainT-Aubin, J., Klein, R. M., \& LANDRY, T. (2005). Age changes in the missing-letter effect revisited. Journal of Experimental Child Psychology, 91, 158-182.

Saint-Aubin, J., Klein, R. M., \& Roy-Charland, A. (2003). Direct assessments of the processing time hypothesis for the missing-letter effect. Journal of Experimental Psychology: Human Perception \& Performance, 29, 1191-1210.
Saint-Aubin, J., \& Poirier, M. (1997). The influence of word function in the missing-letter effect: Further evidence from French. Memory \& Cognition, 25, 666-676.

Schneider, V. I., \& Healy, A. F. (1993). Detecting phonemes and letters in text: Interactions between different types and levels of processes. Memory \& Cognition, 21, 739-751.

Schneider, V. I., Healy, A. F., \& Gesi, A. T. (1991). The role of phonetic processes in letter detection: A reevaluation. Journal of Memory \& Language, 30, 294-318.

\section{NOTE}

1. Twenty-seven native English speakers were tested with the materials used by Greenberg and Tai (2001) in their first experiment. The participants read the same 30 four-sentence paragraphs as in Greenberg and Tai's study, but they did not repeat any sentence aloud before reading the 4-sentence paragraphs. For each target letter $(t$ and $d$ ), omission rate performance was compared for the 5 sentences that were not repeated in Greenberg and Tai's study and the 10 sentences that were repeated in their study. Obviously, here no sentences were repeated. Our results revealed that, for the target letter $d$, there was no difference between repeated and nonrepeated sentences in terms of omissions on the function word and and the control content words. Like Greenberg and Tai, we observed a huge and significant missing-letter effect, with average omission rates of $86 \%$ for and and $18 \%$ for the control content words. For the target letter $t$ in the function word the, our results mirrored those of Greenberg and Tai, who observed a decrease in omission rate from $57.4 \%$ for the nonrepeated sentences to $51.6 \%$ and $53.4 \%$ for the sentences read three and six times, respectively. We observed omission rates of $40.7 \%$ for the target letter $t$ embedded in the nonrepeated sentences and of $33.7 \%$ in the sentences that were repeated in Greenberg and Tai's study (we collapsed together the 5 sentences in the three-readings condition and the 5 sentences in the six-readings condition, as they did in some of their analyses). This difference was significant, as indicated by a planned contrast on the $\left[F(1,52)=5.86, M S_{\mathrm{e}}=114.03\right]$. For the control content words, contrary to Greenberg and Tai (who found a slight increase from $4.1 \%$ omissions for the content words in the single-reading condition to $5.6 \%$ and $7.5 \%$ omissions for the sentences read three and six times, respectively), we found no difference, with $4.4 \%$ and $4.1 \%$ for the sentences in Greenberg and Tai's nonrepeated and repeated conditions, respectively. It is worth mentioning that Greenberg and Tai did not perform separate analyses on the target letters $t$ and $d$. Consequently, it is not possible to know whether or not their small difference in omission rates for content words with the target letter $t$ in the single-reading and multiple-readings conditions was significant.

(Manuscript received July 8, 2005; revision accepted for publication December 22, 2006.) 